\title{
Systematic Proteomics Analysis of Colorectal Cancer: to Recognize Potential Biomarkers and Remedial Target
}

Noosha Zia Jahromi

Islamic Azad University Shahrekord Branch

Amin Mahmoudi Mandani

Islamic Azad University Shahrekord Branch

Nahid Sadeghi Moghadam Kaji

Islamic Azad University Shahrekord Branch

Hadis Abbasi Teshnizi

Islamic Azad University Shahrekord Branch

Atefeh Zamani

Gene Raz Buali

Nasrin Fattahi

Gene Raz Buali

Hossein Sazegar ( $\nabla$ sazegar.hossein59@gmail.com )

Department Biology

Research

Keywords: Systems biology, Proteomics, multilayer, colorectal cancer

Posted Date: October 21st, 2021

DOl: https://doi.org/10.21203/rs.3.rs-965175/v1

License: (c) (1) This work is licensed under a Creative Commons Attribution 4.0 International License. Read Full License 


\section{Abstract}

\section{Background}

Colorectal Cancer (CRC) is a high prevalence malignant of the digestive system. Notwithstanding huge investigation, the precise involved mechanisms are still blunted. It emerges that more research is needed to understand the underlying mechanisms of CRC better. In this investigation, a systems biology method was applied to provide a comprehensive insight through making a multilayer network to recognize novel biomarkers and potential drug targets.

\section{Method}

To identify the affected proteins in this disease, an existing protein expression profile on CRC was attained from ProteomeXchange. In the first step, the quality of the dataset was assessed, and then differentially expressed proteins (DEPs) were recognized based on FDR $<0.05$ as the statistically significant threshold. Subsequently, all identified DEPs were subjected to further enrichment analysis for finding the involved biomedical phenomena. In the following action, IncRNAs and miRNAs related to DEPs were predicted as regulatory layers via Cytoscape to make an integrative network.

\section{Result}

The empirical findings in this study indicated that the identified DEPs were mainly associated with two biomedical terms, such as "Mitochondrial dysfunction" and "Extracellular matrix organization." Additionally, several hub molecules were introduced in the constructed multilayer network, hub-DEPs (ITGB1, ACTG1, ...), hub-miRs (miR-16-5p, miR-26b-5p, ...), and hub-IncRNAs (KCNQ10T1, NEAT1, ...) that Can be deemed as a potential biomarker in the pathogenesis of $\mathrm{CRC}$, further research should concentrate on the investigation of their role in CRC development.

\section{Conclusion}

The attained results of this study may be paving a way to provide a deeper understanding of the vaguely involved mechanisms through an integrative insight into the interaction of proteins with regulatory layers.

\section{Introduction}

Colorectal cancer (CRC) is a well-known non-communicable disease located in the third place of cancer death. In spite of massive investigations on that, its incidence rate is increasing even in developed countries, which could stem from our narrowing knowledge about the exact underlying pathogenesis mechanism of the disorder $(1,2)$. Considering the complicated nature of cancer, focusing on a single molecule or even one biomedical phenomenon is not applicable to decipher exact CRC pathogenesis (3) (4). Owing to the complexity, our understanding is not entirely defined. So that, introducing novel remedial target or early detection biomarkers are potential manners that can shed light on the CRC pathogenicity (5)(6). Systems biology is a promising approach that can provide a holistic insight to 
introduce potential remedial strategies or non-invasive biomarkers to detect this kind of complicated disorder early (7). Nowadays, this technique, alongside the high-throughput data become extensively carried out to recognized novel clues in the disease (8-10). However, far too little attention has been paid to make a holistic insight utilizing the construction of a multilayer network focusing on regulatory layers in the colorectal cancer disease.

Therefore, the object of this study is to re-analyze a proteomics dataset that is coming from comparing the protein expression profile of the tumor and non-tumor tissue to make a multilayer network including differentially expressed proteins and their related regulators such as MicroRNAs and IncRNAs. In order to make a comprehensive view through finding the molecular signature, introducing novel drug target and biomarker alongside the recognition of the pivotal involvement pathways in the pathogenesis of CRC.

\section{Method And Materials}

\section{Data acquisition}

A protein expression profile (PXD019504) related to colorectal cancer patients produced by Tanaka et al. (11) based on a label-free approach was achieved from the ProteomeXchange database (http://proteomecentral.proteomexchange.org) (12). This dataset was including nine tumor tissues from colorectal cancer and nine non-tumor tissues.

\section{Protein Identification process}

To translate the peptide mass spectrum to identified proteins MaxQuant software integrated with Andromeda search engine was used with following parameters: methionine oxidation, N-terminal acetylation, and cysteine carbamidomethyl were chosen as variable and fixed modification, for the following parameter two minimum ratio count was set to quantify the identified proteins, Homo sapiens proteome version of was downloaded as a theoretical spectrum, PSM and protein FDR $<0.05$ was considered as the significant threshold for peptide and protein identification $(13,14)$.

\section{Differentially expressed proteins detection}

To identify differentially expressed proteins among whole identified proteins, Perseus application was applied; after the filtering of the dataset from contaminant and reverse proteins, quality control assessment was carried out employing principal component analysis (PCA), for the next step, three valid value filtering was carried out based on existing groups of the dataset. To find DEPs, a T-test two sample was performed as a statistical method, and permutation-based FDR $<0.05$ with 250 randomizations was considered a significant threshold (15).

\section{GO and Pathway enrichment analysis related DEPs}

To make a conceptual insight into DEPs based on their altered biological function, GO and pathway enrichment analyses were performed through a well-known user-friendly software, Cytoscape (16). 
ClueGO plugin Is a major bioinformatics tool that is used in this study to annotate proteins based on their biological process, molecular function, and cellular component. REVIGO was carried out to condense achieved findings in the form of parent terms. The Reactome database was utilized to understand related biomedical phenomena based on DEPs. In these procedures, adj. $p$-value $<0.05$ was chosen as significant cut-off criteria (17)(18).

\section{Construction of regulatory multilayer network}

In the first step, a protein-protein interaction (PPI) map was constructed by selecting high interaction scores through the STRING database. MirTarBase and miRNet databases were utilized to enrich the constructed PPI, including a significant cut-off to predict related miRNA and IncRNA, respectively (1921). Then, a multilayer network comprised of (DEPs, miRNA, IncRNA) was constructed, which is visualized through Cytoscape software. The constructed network was evaluated based on two common graph theories, like topological parameters and high clustering coefficient, performed by network analysis and MCODE plugin, respectively (22). The criteria for module selection were as follows: MCODE score $\geq$ 2 , degree cut-off $=2$, node score cut-off $=0.2$, max depth $=100$ and $\mathrm{k}$-score $=2$.

\section{Results}

\section{Dataset selection and quality assessment}

According to our consideration of colorectal cancer data selection, two main keywords (colorectal and colon cancer) were used to choose the appropriate dataset; finally, a protein expression profile (PXD 019504) was generated by Tanaka et al. was obtained from the ProteomeXchange database. The selected dataset comprises 18 rectal tissue samples from 9 tumors and nine none-tumors analyzed with a label-free approach. In the first step, to transform the RAW data to the protein knowledge table, MaxQuant software integrated with Andromeda Search engine was applied with the parameters mentioned earlier based on Target decoy strategy. Given the importance of quality control assessment as an indispensable step in omics data analysis (23), the quality control appraisal performed via PCA, a wellknown unsupervised classification approach, can be used to demonstrate the dataset quality. To determine the satisfying quality of the used dataset, case and control clustering are the best sign. It is omitted as an outlier sample concerning the unsatisfying separation of one tumor sample (Figure 1a). To confirm the quality of samples, hieratical clustering was performed, which showed the satisfying quality of the dataset (Figure 1b).

Afterward, all identified proteins were analyzed consistent with FDR $<0.05$ to recognize the differentially expressed proteins (DEPs) using Perseus. According to the selected significant threshold, 1211 DEPs were detected, illustrated by the volcano plot (Figure 2).

\section{Enrichment analyses of DEPs}


To explore the potential biological function of detected DEPs, the GO enrichment analysis was carried out utilizing ClueGO plugin. The obtained results indicate that the alteration in the biological process were considerably related to the metabolic process, more specifically mitochondrial-related pathways, also extracellular matrix organization. Moreover, enriched parent terms in the molecular function category elucidated the involvement of cell adhesion molecule binding, oxidoreductase activity, and NAD binding as the utmost important altered function concerned with DEPs. In addition, changes in the cellular components section were mainly attributed to extracellular exosome, actin cytoskeleton modification like lamellipodia, and focal adhesion. Regarding Reactome pathway analysis, it can be observed that metabolic process pathways, especially mitochondrial-related pathways such as "TCA cycle," Respiratory electron transporter," as well as amino acid metabolism, are the significantly enriched terms that are in line with the above $\mathrm{GO}$ findings. Remarkably, in agreement with the $\mathrm{GO}$ findings, extracellular matrix organization is one of the main enriched terms based on DEPs. (Figure 3, Figure 4)

\section{The analysis of constructed multilayer network}

To detect the key driver of each added regulatory molecule like MiRNA and IncRNA affecting DEPs, a multilayer network comprised of DEPs- predicted MiRNAs and IncRNAs was constructed and was evaluated. Based on the graph theory concept, more specifically the degree parameter, hub molecules of each layer were identified separately.

The $5 \%$ topmost molecule of each layer in respect of Degree was chosen amid 1471 nodes and 8373 edges (Table 1). The most significant proteins with connectivity degree scores were Integrin beta-1 (ITGB1), ribosomal protein S2, mitochondrial (MRPS2), 40S ribosomal protein S20 (RPS20), actin cytoplasmic 2 (ACTG1), Plectin (PLEC), NADH dehydrogenase, mitochondrial (NDUFS8), Paxillin (PXN), Ras-related C3 (RAC1), Vinculin (VCL). In addition to hub proteins, according to degree criteria, hub microRNAs were recognized including miR-16-5p, miR-26b-5p, miR-92a-3p, miR-1-3p, miR-124-3p, miR155-5p, miR-34a-5p, miR-193b-3p, miR-320a, miR-218-5p, since the regulatory role of microRNA they can be deemed as non-invasive biomarker for detection of the disorder. As some of the identified hub MiRNAs were considered as effective regulators in the progression of CRC.

Due to the affection of miRNA expression through IncRNAs function, also in accordance with the crucial role of IncRNAs in transcriptional and post-translational regulation, we were interested in finding the central molecule of this regulatory layer. So, we identified the top node of the IncRNAs layer among 150 curated IncRNAs which is shown in (Table1). Among all identified IncRNAs, KCNQ10T1 was detected as a hub molecule with the highest interaction with miRNA; moreover, NEAT1 and XIST are other predicted IncRNAs in the top-ranked with the highest degree parameter.

Notwithstanding degree criteria, the high clustering coefficient is another indispensable graph theory approach that could be employed to introduce the central driver of biological networks; depending on the mentioned criteria, two modules were recognized in the constructed multilayer network and their related Reactome pathway enrichments identified (Figure 5) 
It is indicated that "mitochondrial related pathways" and "respiratory electron transport" are the essential altered pathways associated with detected modules.

\section{Discussion}

In the face of the advancement of high-throughput approaches and improving the basic knowledge in biological areas, re-analyzing the pre-existed datasets in all sorts of big biological data could be a valuable method to provide a comprehensive insight into underlying mechanisms of the complex disorders. Furthermore, as a trend of biological issues, the construction of a multilayer network could be a valuable assistance in making the best picture of the disease's pathogenesis (8). In the present study, we carried out a re-analysis of a proteomic dataset. After ensuring the quality of the selected dataset, an integrated network was constructed, composed of essential regulatory elements such as predicted MiRNAs and IncRNAs alongside the calculated DEPs. In the next step, through employing well-known systems biology approaches, pivotal biomedical phenomena and key-role player molecules in each added layer were identified probably have a significant role in colorectal cancer development. In accordance with the findings in enrichment analysis, the identified DEPs were mostly enriched in extracellular matrix organization and metabolic process, specifically the mitochondrial related activities like respiratory electron chain and TCA cycle. In agreement with the above findings, the identified hub molecules amid DEPs can be divided into two classes which underscored the modification of "extracellular organization" and "mitochondrial dysfunction." According to mentioned pathways, the classified high degree proteins are involved in extracellular matrix organization were included ITGB1, ACTG1, and PLEC. Subsequently, a bunch of evidence has been designated the pivotal role of extracellular modification by modifying ITGB1 and PLEC in the CRC pathogenesis (24).

Moreover, it has been illustrated that ITGB1 is an important linker between the actin cytoskeleton and extracellular matrix $(25,26)$. Notably, our findings also revealed that ACTG1 is a considerable detected central protein, one of the prominent elements of the actin cytoskeleton known as a key role player in cancer development. Taken together, in line with the previous investigation coupled with considering the blunted pathogenesis mechanisms of colorectal cancer (27), the mediator elements of the extracellular matrix and actin cytoskeleton could be a lost piece of colorectal pathogenesis which further work needs to be done to establish an insight into the relationship between cytoskeleton modification and CRC prognosis.

Interestingly, in agreement with the key role players of extracellular matrix organization in the progression of the disease, cell adhesion molecule binding in molecular function with the highest number of child terms underscore the importance of these biomedical phenomena. Additionally, all the enriched terms in the cellular component in terms of the number of children determined the actin cytoskeleton related terms such as "lamellipodium" and "focal adhesion," which can be described as decisive elements in cancer cell migration and cancer metastasis that is previously explored in the bunch of studies as pivotal factor and potential therapeutic target (28). All in all, during the cancer progression, cell adhesion disorganization is the topmost alteration in metastasis and cellular transformation; despite this valuable finding, the exact 
mechanisms of extracellular matrix elements are still unclear, then deciphering the role of involved elements requires extensive research.

Inconsistent with the previous studies, our results underlined the mitochondrial dysfunction and related metabolic processes such as "TCA cycle" and "Respiratory chain electron transport "as other terms were significantly enriched for the identified DEPs. In this regard, most detected central proteins were concerned with the MRPS isoforms protein as critical protein for mitochondrial functionality. Remarkably, in agreement with the above results, a bundle of recent investigations have focused on the key role player of mitochondrial dysfunction in the pathogenesis of colorectal cancer $(29,30)$. Additionally, in fulfillment of the central findings, the enrichment analysis results underscored the indispensable role of mitochondrial dysfunction and its related metabolic process in the progression of the disorder. Concerning the undeniable fact that cancer is a high energy demand process, such enrichment terms and proteins were predictable. Moreover, in spite of enriched terms about the mitochondrial-related process, we observed other metabolic processes such as "Branched-chain amino acids metabolism" "Fatty acid metabolism," which is noted in preceding colorectal cancer investigations. In this regard, the role of dietary intake of Branched-chain amino acids metabolism in colorectal cancer occurrence has been recently highlighted $(31,32)$.

Involvement of the mentioned biomedical phenomena probably makes a deeper understanding of the underlying pathogenesis mechanisms of colorectal cancer. In the further step of our analysis, we constructed a multilayer network as the main purpose to identify the central molecule among the enriched regulatory layers such as MiRNAs and IncRNAs. The recognized key regulatory elements are listed in table 1.

miRNA is one of the enriched regulatory layers which plays a major role in the pathological aspect of colorectal cancer (33). Considering their regulatory role on protein expression, they can be chosen as a non-invasive biomarker, as indicated in the former investigation as an early detection marker of such complex disorders like colorectal cancer $(34,35)$.

The topmost MiRNA includes miR-16-5p, miR-26b-5p, miR-92a-3p, miR-1-3p, and other detected hub MiRNA, listed in Table1. Remarkably, the majority part of hub miRNAs are indicated as a key-role player of CRC progression. In a recent survey, miR-16-5p was pointed out as a deceive element in tumorigenesis through targeting the SMAD3 in CRC (36). Additionally, Yang Li et al. indicated that miR-26b-5p is a suppressive factor that directly affects FUT4 expression, which in turn regulates metastasis of cancer cells (37).

In accordance with the fact that all identified central molecules have been previously demonstrated as a momentous part in the progression of $\mathrm{CRC}$, it could be described that this regulatory layer should be noted mostly in this kind of complicated diseases as a potential detective biomarker and potential target remedy. 
Another predicted regulatory layer of constructed network is IncRNAs which were shown as an outstanding biomolecular layer that plays a vital role in pre- and post-translational regulations through the impact on proteins and other layers such as miRNAs (38). In spite of the conducted investigations on the regulatory role of IncRNAs in the progression of colorectal cancer, it can be viewed that focusing on single molecules in this sort of layer cannot be applicable to decipher the exact underlying mechanisms of CRC. Regarding the existed bottleneck, in this study, we import predicted IncRNAs in the constructed network based on their regulatory role on the miRNAs to make a holistic insight without a bias to specific IncRNA; through the network analysis, we recognized some topmost IncRNAs via degree parameters; notably, the majority part of detected hub IncRNAs have previously inspected in the progression of CRC. Accordingly, the critical role of KCNQ10T1 in the development of colorectal cancer was determined by inducing aerobic glycolysis, which is designated its proliferative role (39). Interestingly, the pivotal role of NEAT1 was previously explored as a decisive element to the metastasis of cancer cells (40).

Additionally, it is elucidated as a diagnostic biomarker which can be literature validation about our gained findings (41). In agreement with the previous investigations, all identified IncRNAs play a momentous part which led to the development of colorectal cancer through the binding with MiRNAs and proteins $(42,43)$. Notably, amid the central IncRNAs, some of them, such as HELLPAR, DNAAF4-CCPG1 are not yet surveyed on their involvement in CRC.

Furthermore, a proportion of them recently identified in colorectal cancer as DElncRNAs, such as PAX8AS1 which its role is still blunted, and further investigation is needed to decipher its function in the progression of CRC. Notably, between the identified and discussed IncRNAs, PCBP1-AS1 is another regulatory element that is recently noted in other cancer, according to their regulatory role on PRL-3 that is the main marker to the prognosis of colorectal cancer, which is contributed to promoting cell migration and invasion (44). Correspondingly, PCBP1-AS1 could be considered as a decisive element in the disease.

\section{Conclusion}

In conclusion, based on a holistic approach, this study adds a piece of evidence that favors metabolic process involvement, especially mitochondrial dysfunction and its related pathways in the pathogenesis of CRC. In accordance with the constructed multilayer network, which is comprised of (DEPs - MiRNAs IncRNAs), several hub molecules in each layer were introduced like hub-DEPs (ITGB1, ACTG1, ...), hubmiRs (miR-16-5p, miR-26b-5p, ...), and hub-IncRNAs (KCNQ10T1, NEAT1) which are agreed with the previous investigations based on that they are the key factor in the pathogenesis of CRC. Nevertheless, we announced some IncRNAs such as HELLPAR, DNAAF4-CCPG1 which are not explored in the CRC development which could be counted as potential non-invasive biomarkers in the disease. Taken together, all identified findings, more specifically introduced biomarkers, should be investigated in subsequent studies to clarify their role in the vague pathological mechanisms of CRC.

\section{Declarations}


Availability of data and materials

The Datasets analyzed by the current study are available in the PRIDE repository

https://www.ebi.ac.uk/pride/archive/projects/PXD019504

\section{Funding}

Not-applicable

Acknowledgment

None

\section{Author Information}

Noosha Zia Jahromi, Amin Mahmoudi Mandani, Nahid Sadeghi Moghadam Kaji, Hadis Abbasi Teshnizi,, Hossein Sazegar,Dept. of Biology, Faculty of basic sciences, Islamic Azad University, Shahrekord Branch, Shahrekord, Iran

Atefeh Zamani, Nasrin Fattahi, Gene Raz Buali, Genetic and Biotechnology Academy, Isfahan, Iran

\section{Author's contribution}

- Authors contributed equally to this work

- Study supervision by Hossein Sazegar

\section{Ethics declarations}

The Ethics Committee of Islamic Azad University, Shahrekord Branch, Shahrekord, Iran approved the study and waived the need for informed written/verbal consent for this study.

\section{Consent for publication}

Not applicable

\section{Competing interests}

The authors declare that they have no competing interests.

\section{Conflict of interest}

There is no conflict of interest to declare.

human subjects protections 
No human subjects were involved in the project

\section{Clinical relevance statement}

Several announced biological molecules such as IncRNAs and microRNAs can be considered as a potential non-invasive biomarker

\section{References}

1. Rawla P, Sunkara T, Barsouk A. Epidemiology of colorectal cancer: Incidence, mortality, survival, and risk factors. Przeglad Gastroenterologiczny. 2019.

2. Haggar FA, Boushey RP. Colorectal cancer epidemiology: Incidence, mortality, survival, and risk factors. Clin Colon Rectal Surg. 2009;

3. Lindblom A, Zhou XL, Liu T, Liljegren A, Skoglund J, Djureinovic T. Colorectal cancer as a complex disease: Defining at-risk subjects in the general population - A preventive strategy. Expert Review of Anticancer Therapy. 2004.

4. Vega P, Valentín F, Cubiella J. Colorectal cancer diagnosis: Pitfalls and opportunities. World J Gastrointest Oncol. 2015;

5. Nimse SB, Sonawane MD, Song KS, Kim T. Biomarker detection technologies and future directions. Analyst. 2016;

6. Sanjay ST, Fu G, Dou M, Xu F, Liu R, Qi H, et al. Biomarker detection for disease diagnosis using costeffective microfluidic platforms. Analyst. 2015.

7. Ayers D, Day PJ. Systems Medicine: The Application of Systems Biology Approaches for Modern Medical Research and Drug Development. Mol Biol Int. 2015;

8. Subramanian I, Verma S, Kumar S, Jere A, Anamika K. Multi-omics Data Integration, Interpretation, and Its Application. Bioinformatics and Biology Insights. 2020.

9. Kim S, Oesterreich S, Kim S, Park Y, Tseng GC. Integrative clustering of multi-level omics data for disease subtype discovery using sequential double regularization. Biostatistics. 2017;

10. Menyhárt O, Győrffy B. Multi-omics approaches in cancer research with applications in tumor subtyping, prognosis, and diagnosis. Computational and Structural Biotechnology Journal. 2021.

11. Tanaka A, Zhou Y, Shia J, Ginty F, Ogawa M, Klimstra DS, et al. Prolyl 4-hydroxylase alpha 1 protein expression risk-stratifies early stage colorectal cancer. Oncotarget. 2020;

12. Vizcaíno JA, Deutsch EW, Wang R, Csordas A, Reisinger F, Ríos D, et al. ProteomeXchange provides globally coordinated proteomics data submission and dissemination. Nature Biotechnology. 2014. 
13. Tyanova S, Temu T, Cox J. The MaxQuant computational platform for mass spectrometry-based shotgun proteomics. Nat Protoc. 2016;

14. Cox J, Neuhauser N, Michalski A, Scheltema RA, Olsen J V., Mann M. Andromeda: A peptide search engine integrated into the MaxQuant environment. J Proteome Res. 2011;

15. Tyanova S, Temu T, Sinitcyn P, Carlson A, Hein MY, Geiger T, et al. The Perseus computational platform for comprehensive analysis of (prote)omics data. Nature Methods. 2016.

16. Shannon P, Markiel A, Ozier O, Baliga NS, Wang JT, Ramage D, et al. Cytoscape: A software Environment for integrated models of biomolecular interaction networks. Genome Res. 2003;

17. Bindea G, Mlecnik B, Hackl H, Charoentong P, Tosolini M, Kirilovsky A, et al. ClueGO: A Cytoscape plugin to decipher functionally grouped gene ontology and pathway annotation networks. Bioinformatics. 2009;

18. Jassal B, Matthews L, Viteri G, Gong C, Lorente P, Fabregat A, et al. The reactome pathway knowledgebase. Nucleic Acids Res. 2020;

19. Szklarczyk D, Gable AL, Lyon D, Junge A, Wyder S, Huerta-Cepas J, et al. STRING v11: Protein-protein association networks with increased coverage, supporting functional discovery in genome-wide experimental datasets. Nucleic Acids Res. 2019;

20. Huang HY, Lin YCD, Li J, Huang KY, Shrestha S, Hong HC, et al. MiRTarBase 2020: Updates to the experimentally validated microRNA-target interaction database. Nucleic Acids Res. 2020;

21. Chang L, Zhou G, Soufan O, Xia J. miRNet 2.0: Network-based visual analytics for miRNA functional analysis and systems biology. Nucleic Acids Res. 2020;

22. Bandettini WP, Kellman P, Mancini C, Booker OJ, Vasu S, Leung SW, et al. MultiContrast Delayed Enhancement (MCODE) improves detection of subendocardial myocardial infarction by late gadolinium enhancement cardiovascular magnetic resonance: A clinical validation study. J Cardiovasc Magn Reson. 2012;

23. Park T, Kim Y, Kim GT, Kwon MS. Statistical quality control analysis of high-dimensional omics data. Int J Data Min Bioinform. 2017;

24. Liu QZ, Gao XH, Chang WJ, Gong HF, Fu CG, Zhang W, et al. Expression of ITGB1 predicts prognosis in colorectal cancer: A large prospective study based on tissue microarray. Int J Clin Exp Pathol. 2015;

25. Takahashi $\mathrm{K}$. The linkage between $\beta 1$ integrin and the actin cytoskeleton is differentially regulated by tyrosine and serine/threonine phosphorylation of $\beta 1$ integrin in normal and cancerous human breast cells. BMC Cell Biol. 2001; 
26. Yang $X$, Wang S, Yu W, Zheng Y, Wu Y. Inhibition of ITGB1 enhance the anti-tumor effect of cetuximab in colorectal cancer cell. Medicine (Baltimore). 2020;

27. Fleming M, Ravula S, Tatishchev SF, Wang HL. Colorectal carcinoma: Pathologic aspects. Journal of Gastrointestinal Oncology. 2012.

28. Machesky LM. Lamellipodia and filopodia in metastasis and invasion. FEBS Letters. 2008.

29. Burlaka AP, Ganusevich II, Vovk A V., Burlaka AA, Gafurov MR, Lukin SN. Colorectal Cancer and Mitochondrial Dysfunctions of the Adjunct Adipose Tissues: A Case Study. Biomed Res Int. 2018;

30. Lin CS, Liu LT, Ou LH, Pan SC, Lin Cl, Wei YH. Role of mitochondrial function in the invasiveness of human colon cancer cells. Oncol Rep. 2018;

31. Rossi M, Mascaretti F, Parpinel M, Serraino D, Crispo A, Celentano E, et al. Dietary intake of branchedchain amino acids and colorectal cancer risk. Br J Nutr. 2021;

32. Zaytseva Y. Lipid metabolism as a targetable metabolic vulnerability in colorectal cancer. Cancers. 2021.

33. Ding L, Lan Z, Xiong X, Ao H, Feng Y, Gu H, et al. The dual role of microRNAs in colorectal cancer progression. International Journal of Molecular Sciences. 2018.

34. Chen B, Xia Z, Deng YN, Yang Y, Zhang P, Zhu H, et al. Emerging microRNA biomarkers for colorectal cancer diagnosis and prognosis. Open Biology. 2019.

35. Condrat CE, Thompson DC, Barbu MG, Bugnar OL, Boboc A, Cretoiu D, et al. miRNAs as Biomarkers in Disease: Latest Findings Regarding Their Role in Diagnosis and Prognosis. Cells. 2020.

36. Gu Z, Li Z, Xu R, Zhu X, Hu R, Xue Y, et al. miR-16-5p Suppresses Progression and Invasion of Osteosarcoma via Targeting at Smad3. Front Pharmacol. 2020;

37. Li Y, Sun Z, Liu B, Shan Y, Zhao L, Jia L. Tumor-suppressive miR-26a and miR-26b inhibit cell aggressiveness by regulating FUT4 in colorectal cancer. Cell Death Dis. 2017;

38. Siddiqui $\mathrm{H}$, Al-Ghafari A, Choudhry H, Al Doghaither $\mathrm{H}$. Roles of long non-coding RNAs in colorectal cancer tumorigenesis: A review. Mol Clin Oncol. 2019;

39. Chen C, Wei M, Wang C, Sun D, Liu P, Zhong X, et al. Long noncoding RNA KCNQ10T1 promotes colorectal carcinogenesis by enhancing aerobic glycolysis via hexokinase-2. Aging (Albany NY). 2020;

40. Luo Y, Chen JJ, Lv Q, Qin J, Huang YZ, Yu MH, et al. Long non-coding RNA NEAT1 promotes colorectal cancer progression by competitively binding miR-34a with SIRT1 and enhancing the Wnt/ $\beta$-catenin signaling pathway. Cancer Lett. 2019; 
41. Wang Y, Zhang D, Zhang C, Sun Y. The diagnostic and prognostic value of serum IncRNA NEAT1 in colorectal cancer. Cancer Manag Res. 2020;

42. Chen DL, Chen LZ, Lu YX, Zhang DS, Zeng ZL, Pan ZZ, et al. Long noncoding rna xist expedites metastasis and modulates epithelial-mesenchymal transition in colorectal cancer. Cell Death Dis. 2017;

43. Li S, Wu T, Zhang D, Sun X, Zhang X. The long non-coding RNA HCG18 promotes the growth and invasion of colorectal cancer cells through sponging miR-1271 and upregulating MTDH/Wnt/ $\beta$-catenin. Clin Exp Pharmacol Physiol. 2020;

44. Xu H, Zeng Y, Liu L, Gao Q, Jin S, Lan Q, et al. PRL-3 improves colorectal cancer cell proliferation and invasion through IL-8 mediated glycolysis metabolism. Int J Oncol. 2017;

\section{Tables}

Table 1: The identified top central molecules in each layer

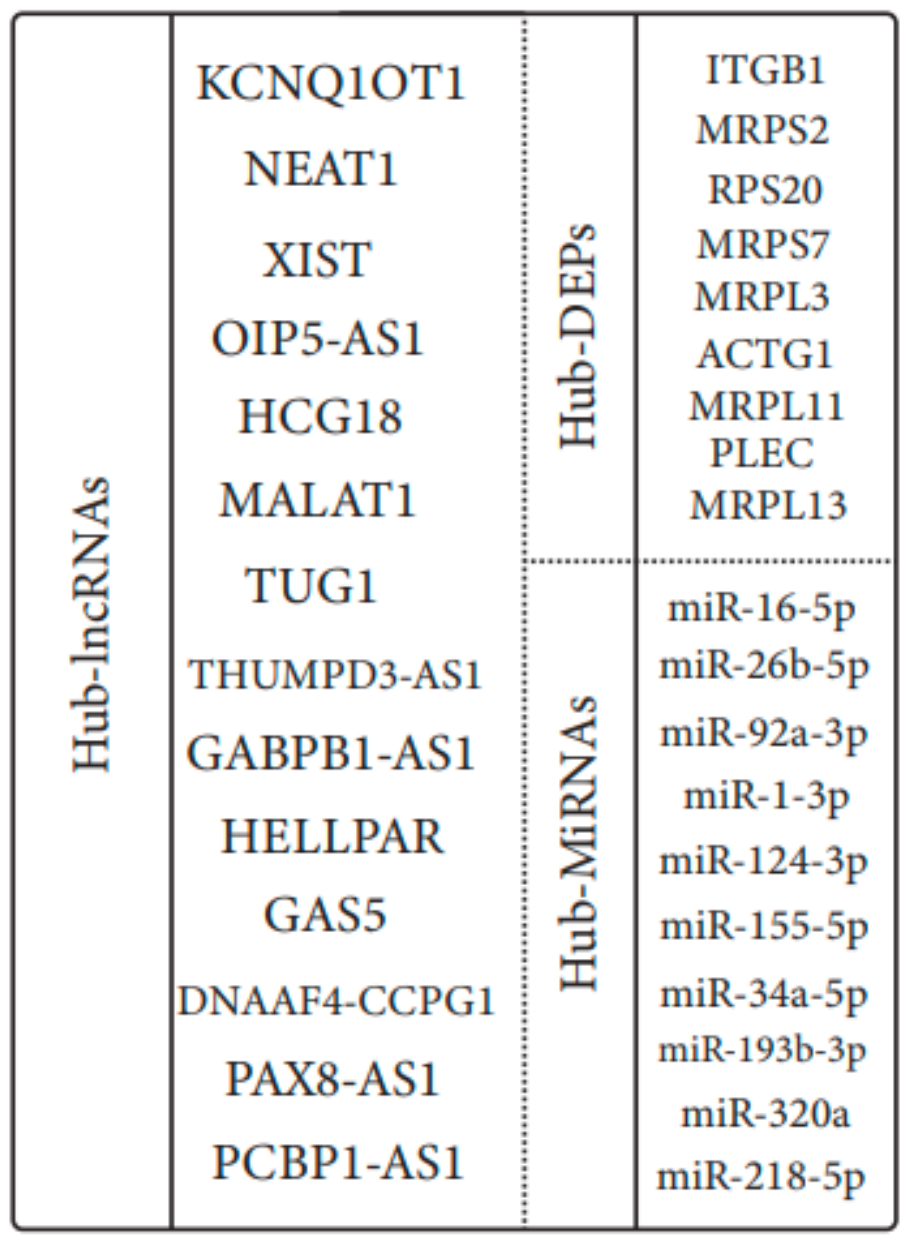

\section{Figures}




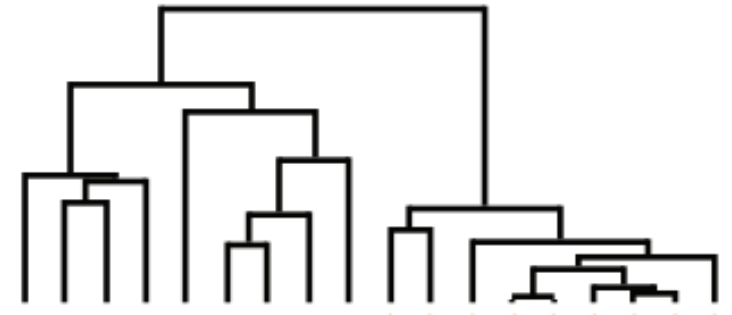

$\square \square \square \square \square \square \square \square \square \triangle A \triangle A \Delta A \Delta A \Delta$
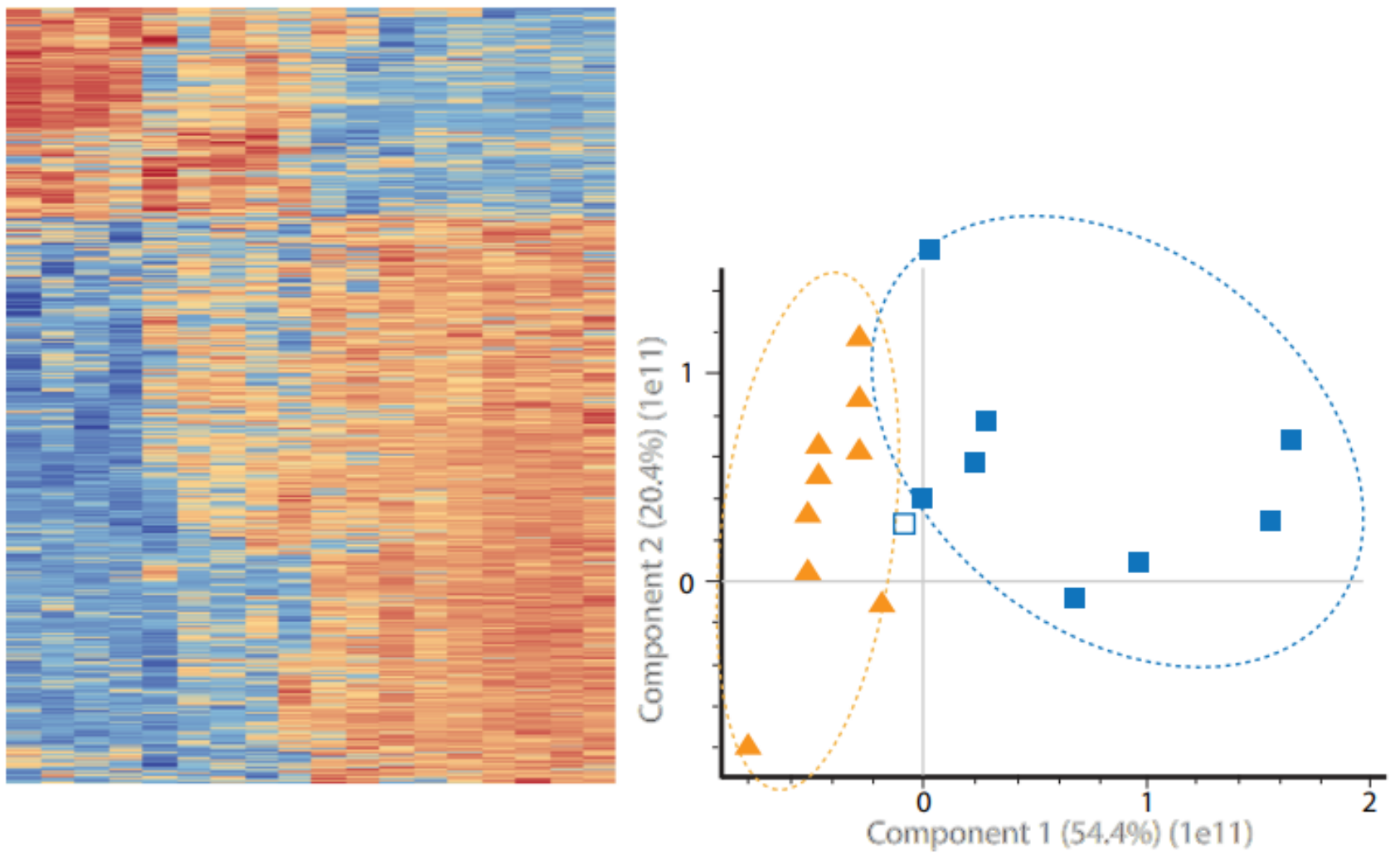

Samples

Tumor $\quad \Delta$ None-Tumor

口Omitted-sample

\section{Figure 1}

Quality control appraisal: To make sure about the quality of the dataset two unsupervised methods were carried out such as A) Principal component analysis (PCA) B) Heatmap. Orange triangle shapes indicate the non-tumor samples, and blue squares are concerned with the tumor samples, also omitted sample was depicted with empty square. 


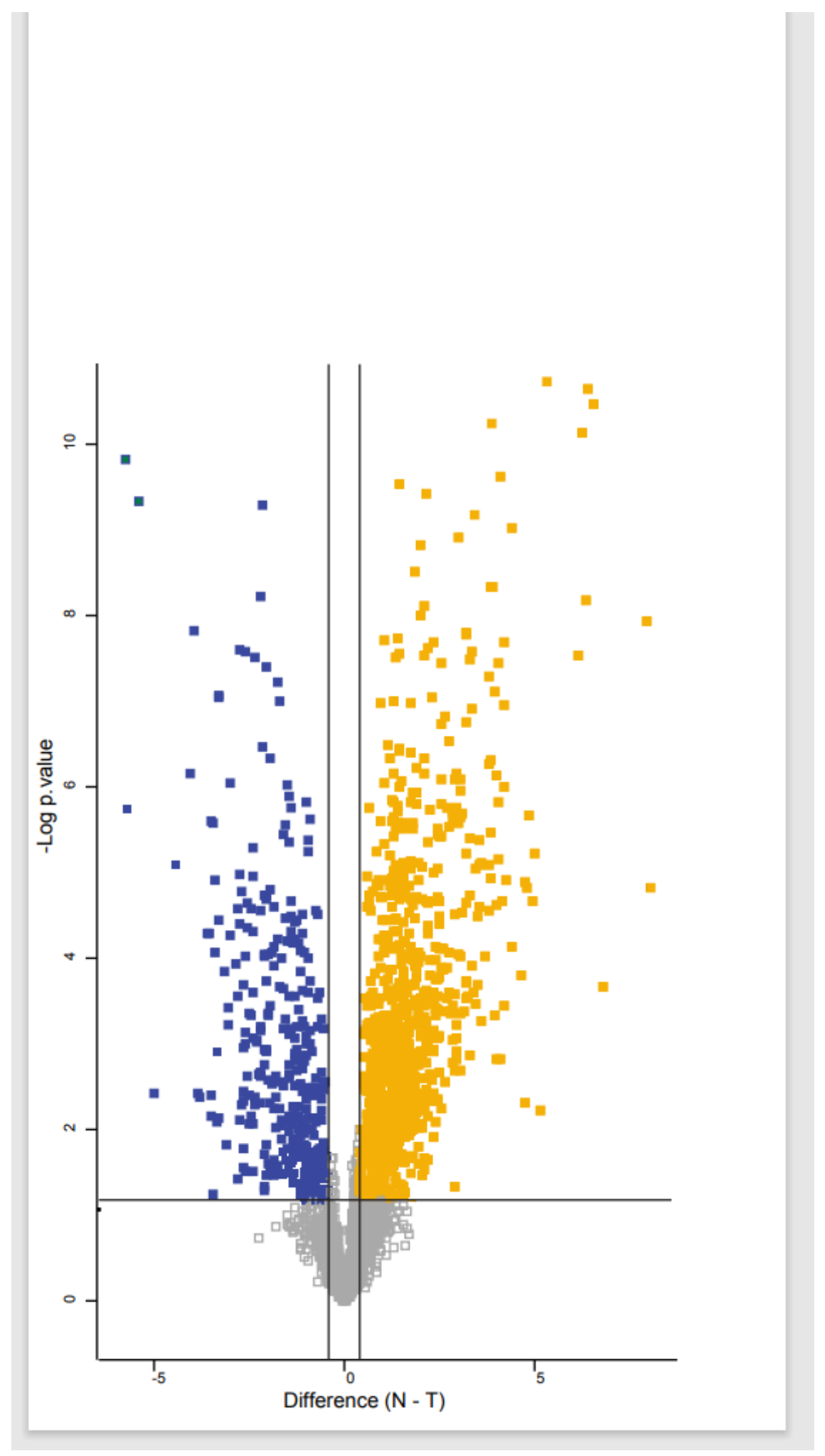

\section{Figure 2}

differentially expressed proteins identification: Volcano plot represents the up-regulated and downregulated proteins. Adj. P-value $<0.05$ was selected as statistically significant threshold. The blue point shows down-regulated proteins, also up-regulated proteins depicted with orange spots. 


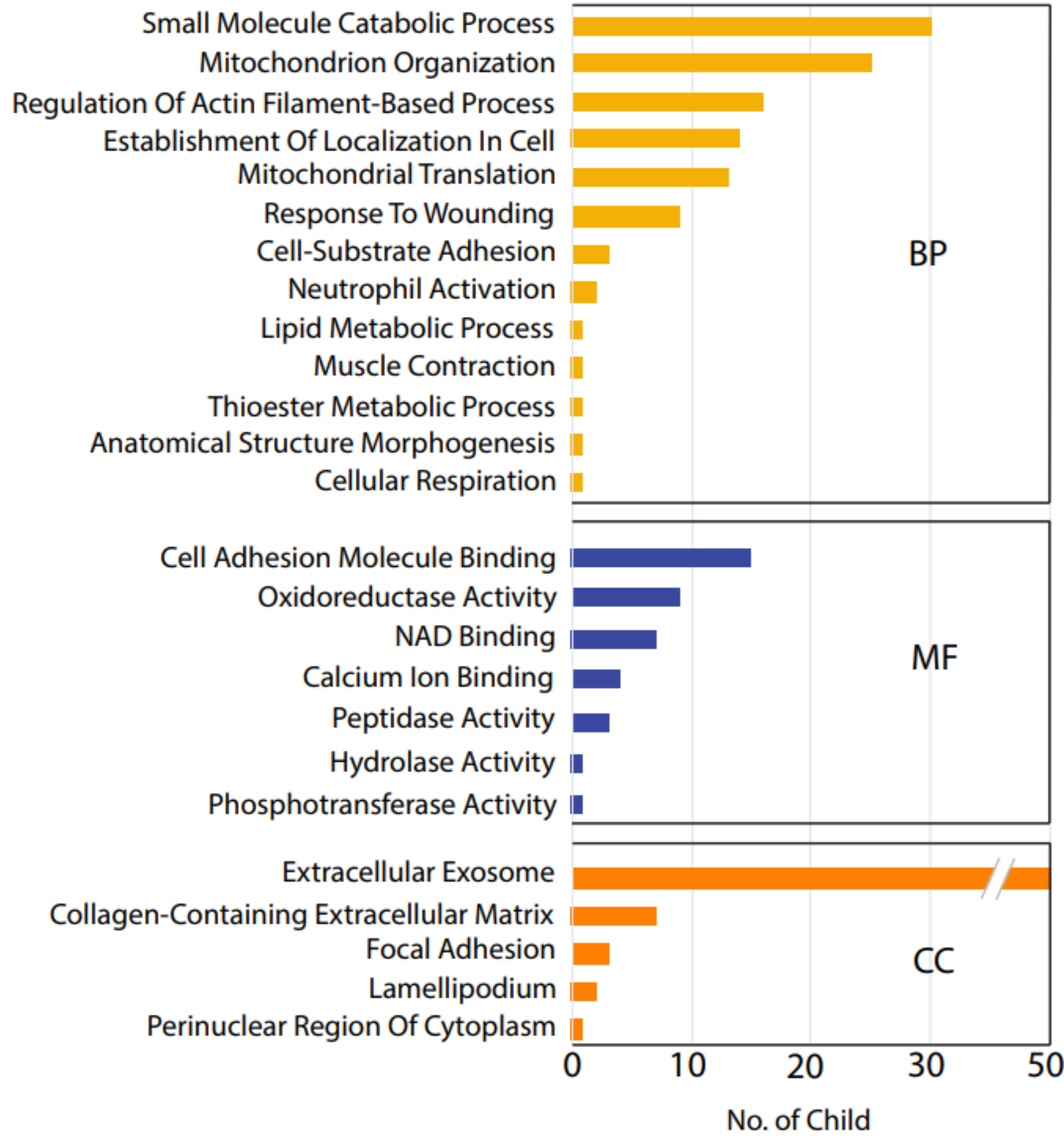

Figure 3

Gene ontology enrichment analysis: GO analysis in three categories were performed concerned with the identified DEPs, the obtained results were summarized as parent terms. The $X$-axis shows the number of child and the $y$-axis indicated the parent terms. Each section was depicted with different colors. 0/05 False discovery rate was considered as a significant threshold 


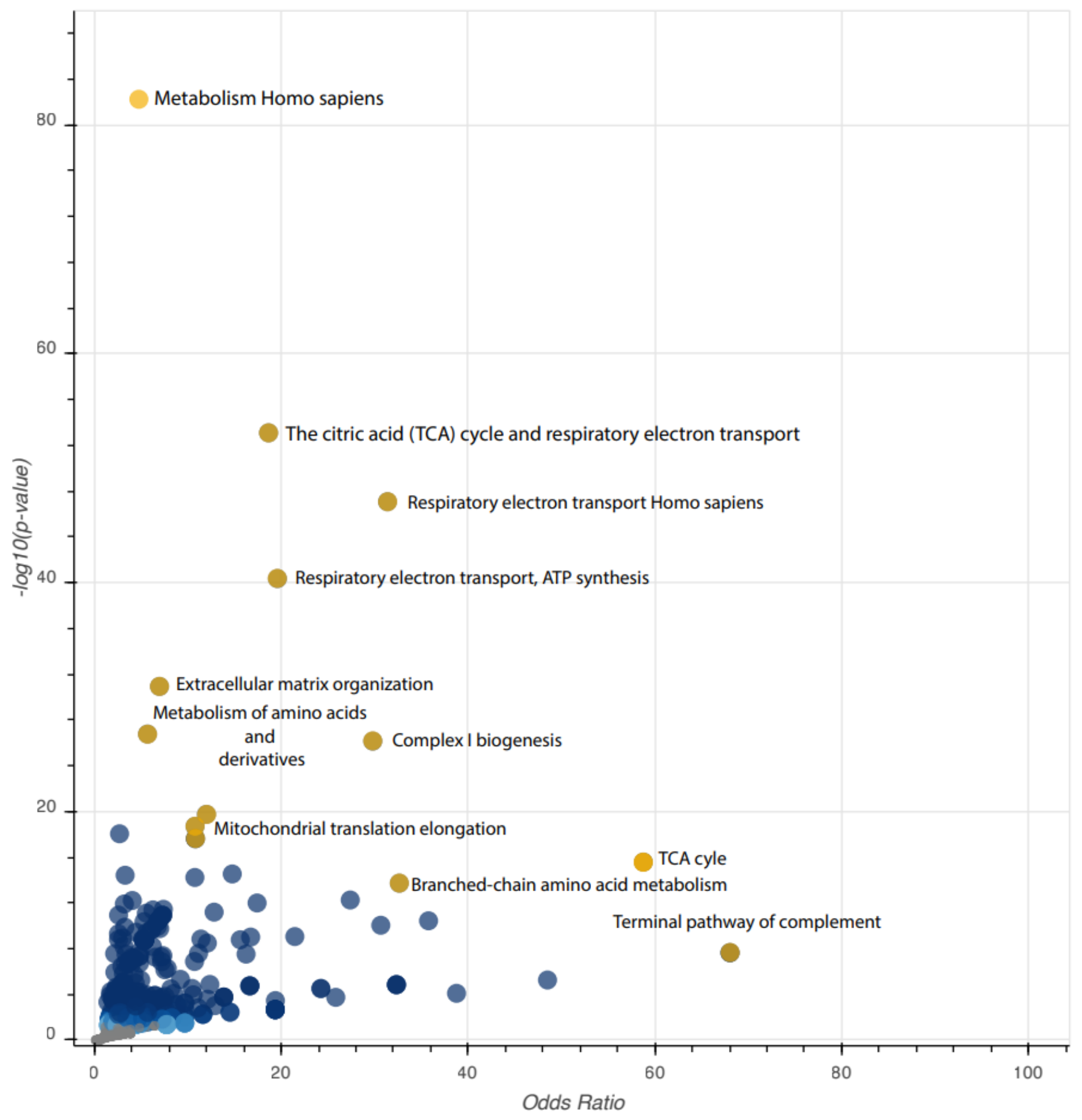

Figure 4

Pathway enrichment analysis was performed on DEPs: the involvement of essential biomedical phenomena was recognized based on altered proteins. The amount of alteration in each pathway was depicted in the x-axis; also, the y-axis shows the selected statistically significant threshold. 


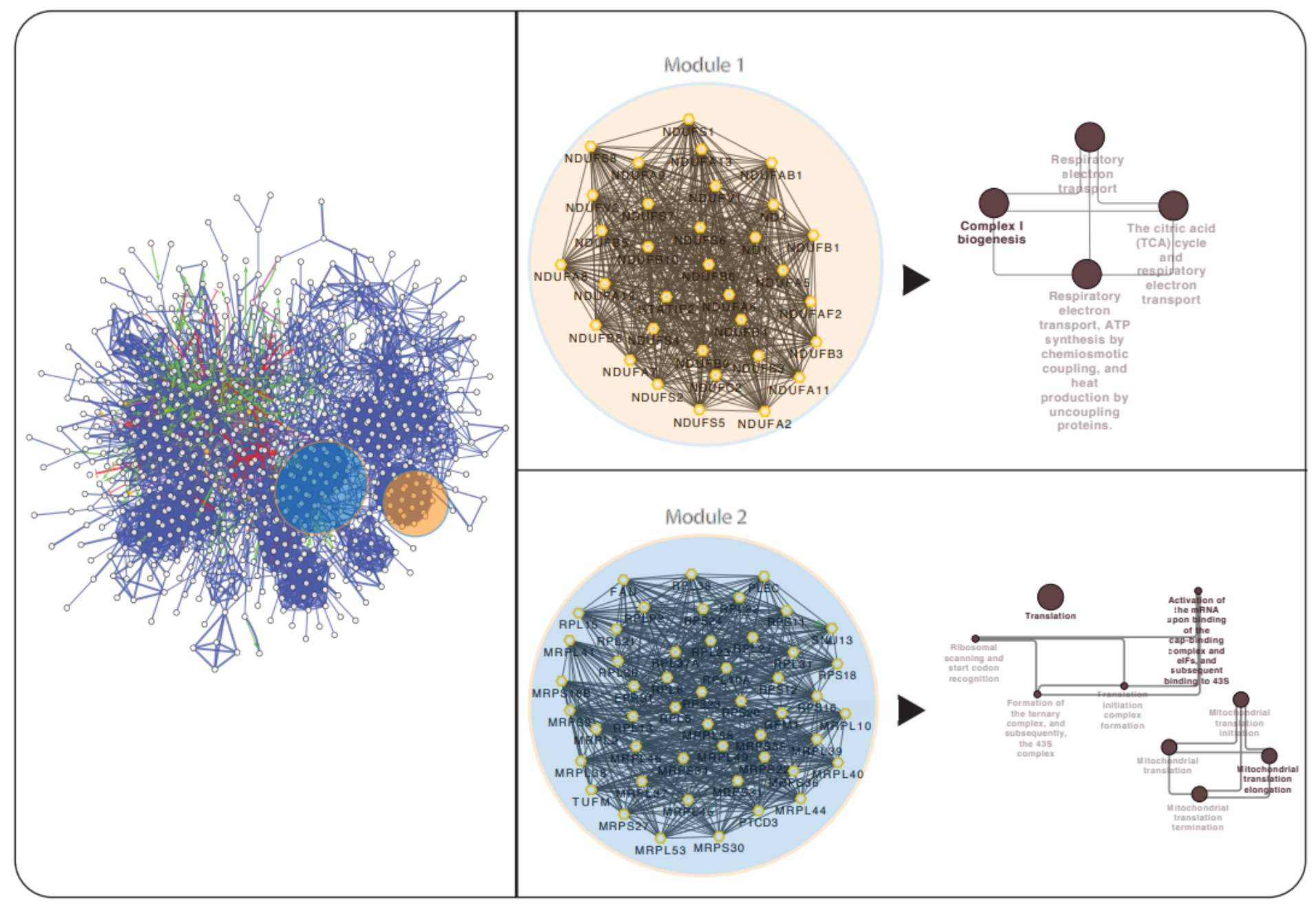

Figure 5

The pathway analysis of two utmost detected modules: The associated pathway of high score detected modules were identified based on STRING database with 0.05 false discovery rate through fisher exact test. 